\title{
EL DESARROLLO REGIONAL Y SUS LIMITANTES: EL CASO DEL CANTÓN DE PURISCAL (COSTA RICA)
}

\author{
ROBERTO FALLAS MORA \\ Centro Universitario de Puriscal \\ Universidad Estatal a Distancia, Costa Rica \\ rfallas@uned.ac.cr \\ FEDERICO QUESADA CHAVES \\ Escuela de Ciencias de la Administración \\ Universidad Estatal a Distancia, Costa Rica \\ fequesada@uned.ac.cr
}

\section{RESUMEN}

Los problemas del desarrollo, dados los procesos de estancamiento de algunas regiones y las asimetrías existentes dentro de los propios países, han sido un tema de relevancia para hilvanar alternativas para las zonas más rezagadas. El estudio se centra en el caso del cantón de Puriscal, que en su momento se consideró un granero de Costa Rica y luego pasó a ser una de las zonas más degradadas del país. El objetivo es describir cómo ocurrió este proceso y dilucidar posibles alternativas de mejora.

PALABRAS CLAVES: DESARROLLO REGIONAL, HETEROGENEIDAD ESTRUCTURAL, CRISIS DEL SECTOR AGRÍCOLA Y AMBIENTAL, POBREZA, PROBLEMÁTICAS SOCIOECONÓMICAS, ALTERNATIVAS DE DESARROLLO.

\section{ABSTRACT}

Puriscal can be characterized as one of the most degraded zones in the country. However, this perception should be analyzed through a historical perspective because, in the past, this same region was known as the Costa Rican barn due to its high basic grains production and productivity. This paper studies the reasons for this zone's deterioration and proposes an eco-development strategy as an alternative to solve its problems. The eco-development concept is based on future generation needs and addresses each specific region socioeconomic and environmental realities. From this concrete reality, and its current inhabitants' concerns, viable alternatives are proposed.
KEYWORDS: REGIONAL DEVELOPMENT, STRUCTURAL HETEROGENITY, ENVIRORMENTAL AND AGRICULTURAL CRISIS, POVERTY, SOCIOECONOMIC PROBLEMS, DEVELOPMENT ALTERNATIVES.

\section{INTRODUCCIÓN}

El cantón de Puriscal pertenece a la provincia de San José, capital de Costa Rica, sin embargo el cantón se fue formando gracias a un proceso de colonización agrícola desde el Valle Central a partir siglo XIX, por lo que la zona ha sido de una fuerte vocación agraria, en especial de productos para el mercado interno, actualmente en una fuerte crisis.

En el estudio, basado en investigación bibliográfica y en entrevistas con líderes de la zona, se describe qué ocasionó el proceso de deterioro ambiental y de rezago en el desarrollo del cantón en estudio y plantea cómo una alternativa de ecodesarrollo podría constituirse en una estrategia de crecimiento sostenido que reanime la economía de la región.

Puriscal se caracteriza por ser una de las zonas más degradadas del país. Sin embargo, hay que analizar su situación desde una perspectiva histórica, ya que en su momento el cantón se tipificaba como el granero de Costa Rica, por su alta producción y productividad en granos básicos.

El concepto de ecodesarrollo al que nos referimos, se basa en el principio de pensar en las necesidades de las generaciones actuales, sin dejar de pensar en las futuras, para lo cual se debe par- 
tir de la realidad socioeconómica y ambiental de cada región específica para plantear alternativas viables, es decir, de la realidad concreta y de la inquietudes de sus pobladores.

Con este propósito, el artículo estudia algunos problemas socioeconómicos de Puriscal, enraizados en un trasfondo vinculado al tema ambiental, el cual está centrado en la degradación de los suelos e implica una serie de consecuencias a todo nivel (Cervantes,1992).

La crisis de la región también se relaciona con el fracaso de diversos intentos de incursión en actividades agrarias, entre ellas cultivos como el tabaco y el café, que por razones externas no lograron consolidarse en el cantón.

Como estrategia para enfrentar los efectos negativos mencionados, se propone retomar el concepto de ecodesarrollo, elaborando alternativas para el desarrollo del cantón, provenientes de las necesidades propuestas y de las inquietudes de las personas en la región, que por lo tanto impliquen metodologías participativas, pero que no pierdan de vista las perspectivas globales.

El caso del cantón de Puriscal no es muy distinto del de otras regiones con problemas de degradación de recursos, y sus demostraciones de desarrollo alternativo deben servir de ejemplo.

La situación puriscaleña no está aislada de la crisis ambiental, la crisis de exclusión, y el abandono paulatino que el modelo de desarrollo neoliberal ha hecho de la producción para el mercado interno y el sector campesino. De manera general, a pesar de los movimientos especulativos, es posible rastrear una tendencia hacia el incremento en los precios de los mismos en el largo plazo (ver las líneas negras).

\section{Objetivo general}

Determinar algunas causas históricas, económicas y ambientales del rezago en el desarrollo del cantón de Puriscal.

\section{Objetivos específicos}

1. Identificar las causas de la crisis ambiental propia del cantón de Puriscal.

2. Describir las problemáticas socioeconómicas fundamentales del cantón.

3. Proponer una estrategia de ecodesarrollo como alternativa de solución a los problemas del cantón.

\section{Relevancia}

A nivel nacional, existen diversos estudios sobre problemas del desarrollo y el mercado laboral, sin embargo en muy pocos se analizan realidades específicas de las regiones. En este sentido, el presente trabajo puede constituirse en un insumo valioso en los procesos de regionalización y desconcentración por los que atraviesa la Universidad Estatal a Distancia (UNED), como base que permita visualizar la meta de una sede que sea un centro cultural y académico que propicie el estudio y la solución de los problemas de su área de influencia.

Por otra parte, el estudio coincide con los objetivos propuestos por la UNED, de promover la investigación y el desarrollo de las regiones más rezagadas del país (UNED, 2011). Hay que considerar que el cantón de Puriscal es de vocación agrícola, con marcados rezagos por la crisis de este sector y que las políticas de apoyo han sido muy limitadas, los proyectos privilegian las regiones Chorotega y Brunca porque acusan los indicadores más graves.

El caso de la inversión de CONARE por medio de los fondos del sistema es otro ejemplo de que Puriscal no recibe inversión pública, las instituciones públicas presentes en la zona tienen un presupuesto muy restringido, según entrevistas con jerarcas. (Guillermo Espinoza, comunicación personal, 1 de setiembre de 2012). Este trabajo puede servir para potenciar esfuerzos de todas las sedes de la UNED y lograr procesos de regionalización más conscientes y con base científica. 


\section{MARCO TEÓRICO}

En el ámbito de la teoría y la política económica, las discusiones sobre la cuestión del desarrollo/ subdesarrollo y su vínculo con el trabajo y las políticas laborales atraviesan por un momento candente, motivado por las políticas neoliberales (hoy en una severa y estructural crisis global) y los procesos de reforma a las leyes laborales que se han presentado casi en todo el mundo, en especial en América Latina (Marín y Vega, 2000).

La carencia de estudios, un inadecuado fundamento teórico y, sobre todo, la correlación de fuerzas actúan contra los intereses de los trabajadores, donde es un hecho que la parte del producto que se adueñan los trabajadores va en detrimento. Este artículo aspira a ser un aporte a estos debates, mediante enfoques de economía crítica y de economía política, tanto de origen clásico como marxista.

En este sentido, se trata de caracterizar la dinámica de las transformaciones económicas y conocer de qué forma y con cuáles resultados se articulan las economías periféricas con los países más desarrollados.. Se plantea también la necesidad de romper el círculo vicioso e inercial de estructuras que perpetúan el rezago y con ello la falta de una generación de empleos de calidad, que particularmente en la zona en estudio se encuentra en un punto muerto (Guillermo Espinoza, comunicación personal, 1 de setiembre de 2012).

En el ámbito académico existe una carencia de investigaciones desde enfoques teóricos críticos que enfaticen lo regional, más bien prevalece la visión neoclásica de la realidad económica y social. También se observa falta de variedad temática, en este sentido desde una perspectiva crítica la actual investigación aporta, a partir del estudio de este cantón, cierta visión alejada de lo que se ha pensado hasta el momento en relación con el vínculo entre crecimiento económico, crisis ambiental, crisis agrícola y la generación de empleos.
No es posible pensar que el crecimiento económico de una región, generará oportunidades de crecimiento y desarrollo, si no va acompañado de una fuerte dosis de interés social pues la dinámica de mercado tiende a eliminar y deteriorar los puestos de trabajo e incrementar la exclusión social.

Las concepciones teóricas y las discusiones sobre el desarrollo y su vínculo con los problemas del trabajo son de vieja data. Podemos citar tres escuelas de pensamiento que adoptan posturas distintas acerca del desarrollo y el trabajo: la teoría neoclásica, la keynesiana y el marxismo. El enfoque de las teorías, más o menos cercanas al marxismo, denominadas en este estudio de economía crítica', analizan la cuestión del desarrollo² y con ello el problema del trabajo, no como un mercado, sino como un espacio de relaciones de poder donde necesariamente, por lo menos en las sociedades del capitalismo histórico, se van a presentar procesos de desarrollo desigual, en los cuales conviven realidades dinámicas y desarrolladas con otras pauperizadas, como parte de una misma dialéctica (Hinkelamert, 1983).

Desde las diversas ópticas planteadas no se ha considerado solo lo que técnicamente significa el salario, el cual posee límites máximos (dados por la productividad) y mínimos (dados por la subsistencia) los cuales son objetivos, sino que en el ámbito del trabajo y las relaciones laborales, constituyen mucho más que un mercado. La estructura laboral es un entramado de relacio-

1. La Teoría Crítica es de inspiración marxista, pero trasciende esta visión teórica. Se consideran aportes como los de la Teoría de la Dependencia, la fuerte crítica a la visión neoliberal del desarrollo de autores como Hinkelamert y Gallardo y los aportes que ha hecho más recientemente la sociología del trabajo a las investigaciones sobre el tema.

2. Se da la existencia del deseado desarrollo en una región o país si efectivamente ocurre una mejora palpable de la calidad de vida humana en dicha región, esto supera en mucho la visión neoclásica que confunde desarrollo con crecimiento económico. 
nes sociales, las cuales están regidas por hechos de poder (Duchrow y Hinkelammert, 2004).

Las relaciones están determinadas por correlaciones de fuerzas que poseen un carácter histórico y que determinan el que existan, perpetuados estructuralmente y con una lógica inercial, lugares geográfico-sociales desarrollados que tienden a permanecer en este estado, al mismo tiempo que ocurre la existencia de la pauperización, lo cual se refleja en niveles de productividad pobres, con su respectivo impacto en los salarios. En esta dirección las dinámicas económicas, tecnológicas y políticas entran en juego, un ejemplo es la nueva tendencia global de "crecimiento intensivo de la economía, con un ínfimo crecimiento de empleo" (Duchrow y Hinkelammert, 2004).

En este contexto, las luchas de poder se materializan en sentido político, por ejemplo, en la legislación laboral y los controles y efectividad de tal legislación que debe garantizar el Estado, pero eso es reflejo de variables más de tipo económico: ¿quiénes y en cuáles condiciones controlan los medios de producción?, ¿cómo se imponen los salarios y las condiciones laborales?, ¿cómo contrapesa la organización de los trabajadores y se posibilita/imposibilita su organización? (Linares, 2006).

En el engranaje coexisten elementos ideológicos y culturales que poseen importancia, por ende, la problemática del trabajo estaría plenamente vinculada a las condiciones y los niveles de desarrollo y a las lógicas que todo ello propicia (Linares, 2006).

Los aportes de la teoría crítica permiten además tener una visión totalizadora y más amplia, la cual vincula dos grandes temas de la actualidad; definidos por las dinámicas del desarrollo y su vínculo con las condiciones actuales del mundo del trabajo.

Por su parte, la visión neoclásica sobre el mercado de trabajo, la cual impera actualmente, no brinda explicaciones adecuadas sobre los desequilibrios persistentes en el mercado laboral, sobre todo por el hecho de tratar el trabajo como una mercancía común y corriente, lo cual es muy limitante y ha inspirado políticas de empleo poco eficaces, basadas en la flexibilización en cuanto al uso de la fuerza de trabajo y en la reducción de los costos laborales directos e indirectos, cuyos resultados han sido dramáticos. Lo anterior obliga a revisar sus postulados, su diagnóstico y sus recomendaciones (Neffa, 2006).

Los postulados neoclásicos han logrado impulsar e implementar en Costa Rica un modelo de desarrollo que es incapaz de atender las necesidades propias de las regiones y que, en nombre de la eficiencia y las llamadas ventajas comparativas, ha golpeado fuertemente al sector agrícola, como es el caso del cantó en estudio. El sector se debilita y muchas familias campesinas se sumen en la pobreza pues por su limitada preparación no cuentan con buenas alternativas de empleo en la zona, que tampoco se generan por las dinámicas propias de este modelo, y por otras circunstancias de alcance histórico y estructural propias del cantón y que será necesario dilucidar.

El cantón de Puriscal y en general la subregión investigada se han visto afectados por las dinámicas del desarrollo desigual, además se encuentran en polos geográficos y sociales de precarización y pauperización, pues ninguno de los ejes dinámicos como turismo, agricultura de exportación e inversión extranjera directa, tienen impacto real en la zona, la cual se encuentran en estado de abandono por parte del Estado. Sumado a lo anterior, el modelo de desarrollo seguido por Costa Rica, a pauperizado enormemente la producción para el mercado interno, a la suerte de la competencia global.

Una definición muy importante para entender estas dinámicas en el capitalismo actual es el concepto de heterogeneidad estructural, concepto acuñado por la CEPAL y que se refiere al hecho de que en al capitalismo subdesarrollado, 
conviven sectores muy dinámicos y modernizados (en el caso de Costa Rica la llamada nueva economía definida por el sector financiero, exportador, de IED y turismo), localizados social y geográficamente junto a sectores más atrasados y pauperizados (en este caso la economía campesina, los grupos indígenas, muchas de las Pymes y todo lo que se conoce como sector informal). Lo anterior crea dinámicas de desarrollo desigual, muchas veces potenciadas por el modelo de desarrollo (en nuestro caso el impacto adverso del aperturismo indiscriminado y el abandono del sector campesino con la eliminación del Consejo Nacional de Producción (CNP) y el debilitamiento del Ministerio de Agricultura y Ganadería (MAG) (Vargas, L. P, comunicación personal, 10 de octubre de 2009).

\section{MÉTODOLOGÍA Recolección de la información}

En un primer momento la investigación se centró en la revisión de fuentes bibliográficas secundarias disponibles en los diversos centros de documentación en el cantón, en bibliotecas y en Internet, que dieran cuenta de los fundamentos teóricos conceptuales para interpretar las transformaciones estructurales desarrolladas durante los últimas dos décadas en el cantón en estudio, contextualizándolas dentro de la realidad nacional y global. Se describió la causa fundamental de los problemas del cantón y se cuantificó.

También se realizó una revisión bibliográfica de fuentes secundarias tales como el Censo del 2010 del Instituto Nacional de Estadística y Censo (INEC), la Encuesta de Hogares, el Índice de Desarrollo Cantonal generado por el Programa de Naciones Unidas para el Desarrollo (PNUD) y la Caja Costarricense de Seguro Social (CCSS).

Con el fin de concretar la información obtenida en la realidad cantonal, se realizaron una serie de entrevistas a líderes de la zona.

\section{Alcances y limitaciones}

Este trabajo se limitó al área de influencia inmediata del Centro Universitario de la UNED en Puriscal. El poco tiempo y la falta de recursos en general, limitaron la labor de recolección de datos y el logro de los objetivos. El proceso de investigación inició con la realización de un foro sobre desarrollo regional, por parte del Centro de Investigación en Cultura y Desarrollo (CICDE) de la UNED.

\section{Causas profundas y raíz ambiental de la crisis en Puriscal}

Montoya (2004) retomó la perspectiva de la antropóloga Peggy F. Barlett, quien realizó estudios en los años 70 sobre el modo y las condiciones de vida de los campesinos en Puriscal. Pese a los logros y los aires de progreso que se respiraban en la época, la Dra. Barlett logró visualizar una serie de problemas a futuro, los cuales se han venido cumpliendo. Problemas relacionados con la sostenibilidad del modelo agrícola y las condiciones del cantón para darle sustento a su población creciente.

El cantón de Puriscal, aparte de su riqueza histórica colonial y precolombina, gracias a la cual perviven en la zona dos territorios indígenas (Quitirrisí y Zapatón), es producto del proceso de colonización agrícola de Costa Rica, que surgió desde el Valle Central a mediados del Siglo XIX, debido a una dinámica en la cual la degradación de los recursos naturales condujo a la expulsión de población sobrante, que no pudo apropiarse de las tierras más ricas y encarecidas del área central del país, por lo cual emigró a la periferia, en este caso hacia el que sería el futuro cantón de Puriscal, en busca de tierras para expandir el cultivo del café, granos básicos, caña, ganadería extensiva y en la segunda del siglo XX el tabaco (Somarribas y Patterson, 1997). 
El proceso histórico que paulatinamente generó el agotamiento del suelo en el cantón se relaciona con el hecho de que el Estado daba incentivos para que en la montaña se fueran abriendo las abras, lo que aunado a las fuertes pendientes, las prácticas agrícolas y ganaderas no acordes con la vocación forestal del suelo provocaron su degradación ambiental, afectándolo cual afectó la productividad agropecuaria y aumentó el uso de insumos para la producción (Cervantes, 1992).

La situación descrita empeoró por una serie de circunstancias históricas y económicas más recientes: la penetración de la industria de los lácteos destruyó la producción campesina de leche, la poca productividad del tabaco y sus mismas consecuencias ambientales sucumbieron ante la competencia externa por la baja en los aranceles, los bajos precios del café y la crítica situación de los granos básicos agravaron el abandono paulatino del sector campesino, el cual quedó desprotegido ante el auge del sector exportador.

La suma de los factores descritos generaró la pobreza y la poca producción en el cantón, lo que produjo graves consecuencias socioeconómicas, que se manifiestaron en tasas de emigración negativas desde los años 50 del siglo XX, debido la falencia grave del cantón de no estar vinculado a ningún eje dinámico del nuevo modelo de desarrollo (turismo, inversión extranjera directa y exportaciones).

Otro elemento crítico para el cantón es la miopía de tantos líderes políticos y generaciones enteras de destacados profesionales, quienes han servido al desarrollo de otros cantones por falta de opciones locales. Actualmente, la existencia de un mercado laboral precario e insuficiente ha convertido a Santiago de Puriscal y sus alrededores en una ciudad dormitorio. Esta situación que fue promovida por conveniencia por algunos empresarios del cantón, minó las posibilida- des de desarrollo local, primordialmente porque es la gente joven en edad de trabajar y la más estudiada la primera que emigra del cantón, profundizando la pobreza y la falta de desarrollo.

El modelo de desarrollo actual del país proporciona apoyo a los grandes monocultivos mecanizados y ha dejado en total abandono a los pequeños campesinos que son los que predominan en el cantón. Existe una dependencia a los insumos externos, de alto precio y del mercado internacional, que ha resultado ser ruinosa para los campesinos, que últimamente no siembran porque la actividad solo acarrea pérdidas.

En este contexto es normal que las familias busquen opciones de subsistencia fuera del cantón (en otros sectores) para poder subsistir o que Santiago y las poblaciones aledañas se vayan acercando cada vez más al fenómeno de ciudad dormitorio. Así las cosas, la amenaza de la insostenibilidad del crecimiento poblacional se ha visto resuelta mediante una diáspora (emigración interna) puriscaleña que no ha permitido el crecimiento de la población. Las opciones de industrias o cultivos que solventen alternativas no han tenido éxito, como en su momento lo fue el tabaco o el café.

Sin embargo, los campesinos han buscado formas de vincularse al mercado externo, con poco apoyo del Estado, porque en el cantón se ha trabajado, a nivel experimental, con varias opciones que podrían ser una buena alternativa, entre ellas una planta para producir biocombustibles y la acerola (fruta tropical).

Para el desarrollo de estos cultivos, es necesaria la investigación de las universidades públicas, que propongan opciones viables para la población, sin embargo como las áreas costeras y fronterizas se encuentran en condiciones aún más graves en cuanto a indicadores sociales y de desarrollo, los esfuerzos se destinan a esas zonas, en especial los fondos de Regionalización del Consejo Nacional de Rectores (CONARE). 
FIGURA 1

ÍNDICE DE PRECIOS DE LOS ALIMENTOS
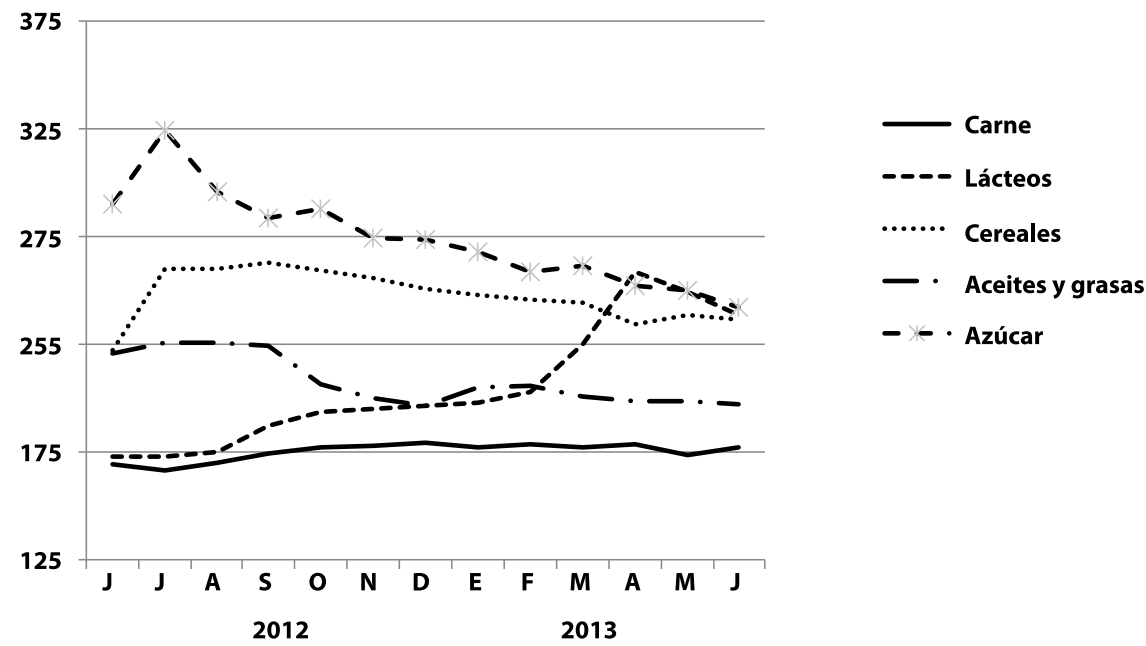

Fuente: Organización de las Naciones Unidas para la Alimentación y la agricultura.

Como se muestra en el figuras 1 y 2, las tendencias descritas se ven contrarrestadas, en parte, por un nuevo autor global: la crisis alimentaria, que en cierta forma surge como una esperanza para los campesinos, ya que de nuevo se visualiza la posibilidad de promover la producción agrícola de alimentos y la autosuficiencia alimentaria. Si se observa la figura 1, durante el año 2008 hasta la fecha, los precios de los alimentos han subido debido a movimientos especulativos de distintas naturalezas, lo cual se refleja especialmente en la coyuntura económica ejemplificada en la figura 2. Esta situación podría ser una oportunidad, si existiera una base agraria sólida en el cantón, así como también condiciones logísticas favorables.

Hay una nueva situación global, que en cierta forma es una esperanza para el sector campesino, relacionada con el aumento paulatino del precio de los alimentos, lo que advierte a los gobiernos acerca del terrible descuido de la autosuficiencia alimentaria y del sector campesino. Esta misma situación se aprecia en el figura 2.

FIGURA 2

INCREMENTO DE ÍNDICE DE PRECIOS DE LOS ALIMENTOS

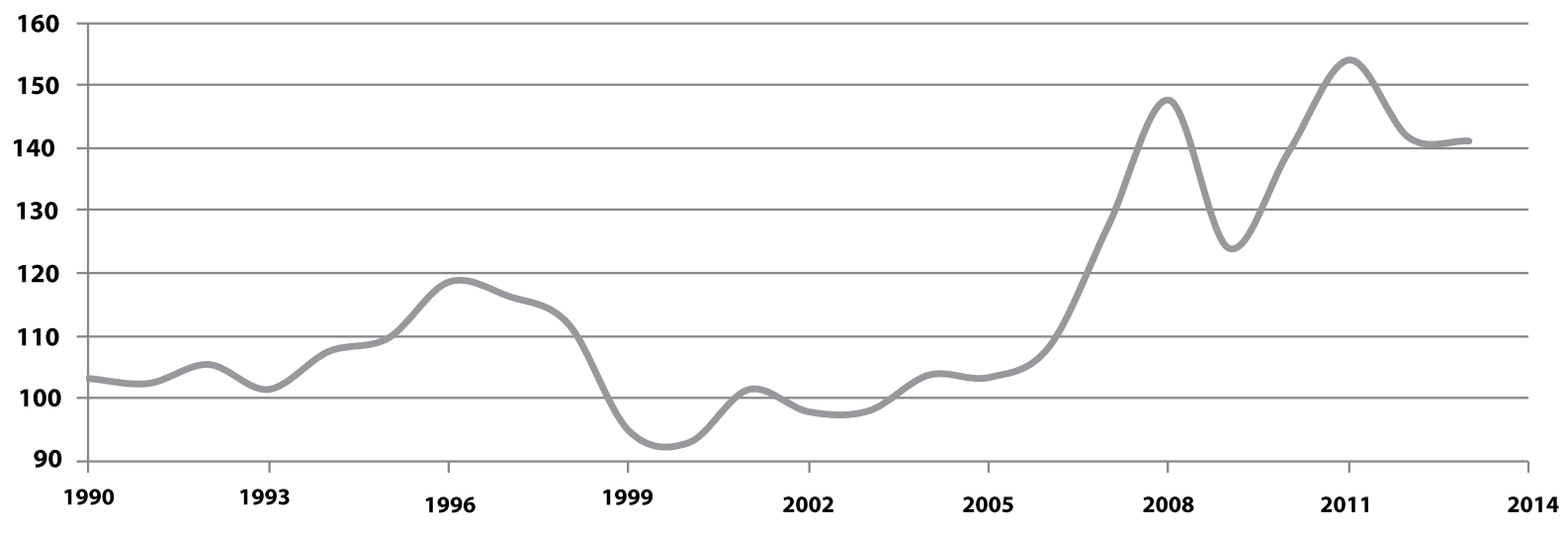

Fuente: Organización de las Naciones Unidas para la Alimentación y la agricultura. 
FIGURA 3

DESARROLLO HUMANO COMPARADO (TALAMANCA, PURISCAL Y SANTA ANA)
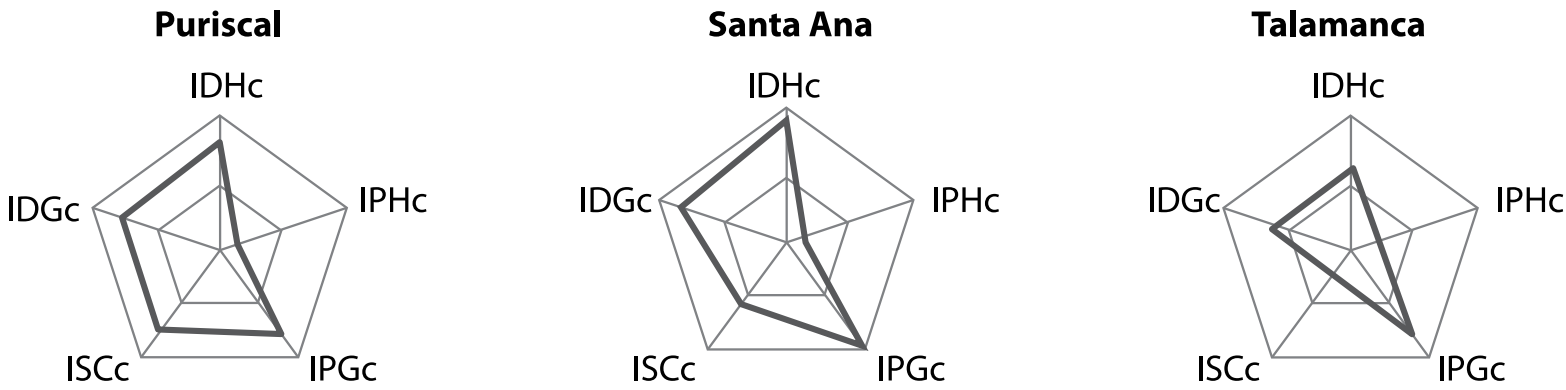

Fuevie: Elaboración propia con base en datos del Programa de Desarrollo Humano para las Naciones Unidas (PNUD, 2009).

Nota. IDH: Índice de desarrollo humano; IDHC: Índice de desarrollo humano cantonal; IDGc: Índice de desarrollo humano relativo al género; ISCc: Índice de seguridad ciudadana cantonal; IPGc: Índice de potenciación del género cantonal; IPHc: Índice de pobreza humana cantonal.

TABLA 1

FICHA TÉCNICA DE PURISCAL, INDICADORES DE DESARROLLO HUMANO

\begin{tabular}{|c|c|c|c|c|c|}
\hline \multicolumn{2}{|l|}{ Cantón: 104 Puriscal } & & & \multicolumn{2}{|c|}{ IDHc } \\
\hline $\begin{array}{l}\text { Extensión territorial }\left(\mathrm{Km}^{2}\right) \text { : } \\
\text { (Entre paréntesis se indica la posición del cantón } \\
\text { con respecto al total de cantones) }\end{array}$ & 554,0 & & & $\mathrm{ISCc}$ & \\
\hline \multirow{2}{*}{ Índice ó Indicador } & \multicolumn{5}{|c|}{ Año } \\
\hline & 2007 & 2008 & 2009 & 2010 & 2011 \\
\hline IDHC & $0,807(17)$ & $0,807(21)$ & $0,804(26)$ & $0,800(28)$ & $0,784(38)$ \\
\hline IEVc & $0,916(3)$ & $0,924(7)$ & $0,909(7)$ & $0,879(11)$ & $0,885(21)$ \\
\hline Esperanza de vida (años) & 82,1 & 82,3 & 81,8 & 80,8 & 81,0 \\
\hline $\mathrm{ICC}$ & $0,950(5)$ & $0,936(8)$ & $0,950(10)$ & $0,950(12)$ & $0,891(36)$ \\
\hline Tasa de matriculación primaria (\%) & 93,0 & 91,5 & 89,8 & 88,7 & 87,3 \\
\hline Tasa de matriculación secundaria (\%) & 100,0 & 97,2 & 100,0 & 100,0 & 88,0 \\
\hline Tasa de matriculación global (\%) & 98,3 & 95,8 & 97,4 & 97,2 & 87,9 \\
\hline Tasa de alfabetización adulta (\%) & 94,3 & 94,6 & 95,0 & 95,4 & 95,7 \\
\hline IBMC & $0,554(51)$ & $0,560(51)$ & $0,553(51)$ & $0,569(51)$ & $0,577(50)$ \\
\hline Consumo per cápita de electricidad (Kwh) & 637,5 & 644,3 & 636,6 & 654,6 & 663,9 \\
\hline IPHC & $10,007(5)$ & $10,063(4)$ & $10,810(3)$ & $13,525(30)$ & $13,605(28)$ \\
\hline IPGC & $0,777(39)$ & $0,788(39)$ & $0,776(44)$ & $0,748(65)$ & $0,753(61)$ \\
\hline IDGC & $0,768(11)$ & $0,764(13)$ & $0,766(16)$ & $0,758(19)$ & $0,743(32)$ \\
\hline Población (habitantes) -estimación- & 31053 & 31154 & 31205 & 31207 & 31189 \\
\hline
\end{tabular}

Fuente: Programa de Desarrollo Humano para las Naciones Unidas (PNUD, 2009). 
La subregión en estudio presenta altos índices de pobreza y un desarrollo humano medio en relación con el resto del país. Según el Índice de Desarrollo Humano Cantonal de 2011 (PNUD y UCR, 2011), Puriscal se ubica en el puesto $26^{3}$, lo que indica que pese a la problemática en estudio, los indicadores de desarrollo del cantón no son tan bajos, como se podría esperar ante el rezago en el desarrollo (ver tabla 1).

Las brechas entre el desarrollo humano de los cantones del país van en aumento, en el caso de Puriscal porque esta zona no ha sido dinamizada por ninguno de los nuevos ejes de desarrollo capitalista (Hikelamert, 1983), como son el turismo, la agro exportación no tradicional y la gran inversión extranjera, por lo que el rezago se hace evidente y se manifiesta, por ejemplo, en el hecho de haberse convertido en una ciudad dormitorio, o sea un lugar donde mucha gente pasa la noche, para estudiar y trabajar en San José.

Esta problemática se manifiesta en la crisis del sector agrícola, que si bien es un fenómeno global, aquí adquiere rasgos particulares y de mayor virulencia, entre otras razones por la pendiente del terreno. Todo esto se asocia a otros fenómenos del desarrollo económico y del mercado laboral en la zona: la emigración y la poca generación de empleo que produzca movilidad social en el cantón.

Si bien los problemas vinculados con las dinámicas de desarrollo, la crisis agrícola y el mercado laboral constituyen temas recurrentes en algunas investigaciones, los estudios de caso a nivel regional son escasos en Costa Rica.

3. En cuanto al desarrollo humano, los cantones peor ubicados han sido históricamente Alajuelita y Talamanca, mientras que los cantones mejor ubicados han sido Montes de Oca y Santa Ana.
En Puriscal podemos visualizar que se registran tendencias y problemáticas palpables, a nivel de las sociedades capitalistas, tal como el conocido desarrollo desigual, la llamada heterogeneidad estructural que tiende a generar, zonas rezagadas, con una localización a nivel social y geográfico determinadas, las cuales en este caso en particular se profundizan (Vargas, L. P, comunicación personal, 10 de octubre de 2009).

\section{Estructura productiva y tendencias del cantón de Puriscal}

La producción en Puriscal continúa siendo de base agrícola para el mercado interno que, por otra parte, ha sido el sector más afectado con las políticas económicas neoliberales y del cual se espera recibirá impactos más fuertes con la profundización del modelo aperturista, quizá el caso que más ilustra esto fue la apertura unilateral en el sector tabacalero, la cual destruyó su producción en la zona.

Los nuevos empleos creados, por lo general de carácter informal, son de tipo urbano (en las cabeceras de los cantones), totalmente insuficiente y comprenden básicamente el ámbito de los servicios. Los intentos por vincular a la región con proyectos agroexportadores han sido infructuosos por razones diversas que deberán ser motivo de futuras investigaciones. 
TABLA 2

\section{MICRO, PEQUEÑA Y GRAN EMPRESA EN PURISCAL (PERIODO 2003-2008)}

\begin{tabular}{|l|c|c|c|c|c|c|}
\hline \multicolumn{1}{|c|}{ Año } & 2003 & 2004 & 2005 & 2006 & 2007 & 2008 \\
\hline $\begin{array}{l}\text { Micro empresas } \\
\text { comerciales }\end{array}$ & 272 & 60 & 53 & 48 & 63 & 73 \\
\hline $\begin{array}{l}\text { Micro empresas } \\
\text { de servicios }\end{array}$ & 80 & 71 & 67 & 65 & 72 & 74 \\
\hline $\begin{array}{l}\text { Micro empresas } \\
\text { industriales }\end{array}$ & 16 & 17 & 14 & 10 & 10 & 12 \\
\hline $\begin{array}{l}\text { Pequeñas empresas } \\
\text { comerciales }\end{array}$ & 9 & 9 & 10 & 9 & 8 & 9 \\
\hline $\begin{array}{l}\text { Pequeñas empresas } \\
\text { de servicios }\end{array}$ & 11 & 15 & 11 & 18 & 22 & 22 \\
\hline $\begin{array}{l}\text { Pequeñas empresas } \\
\text { industriales }\end{array}$ & 4 & 5 & 4 & 2 & 3 & 3 \\
\hline $\begin{array}{l}\text { Grandes empresas } \\
\text { comerciales }\end{array}$ & 1 & 1 & n.d. & 1 & 1 & 1 \\
\hline $\begin{array}{l}\text { Grandes empresas } \\
\text { de servicios }\end{array}$ & 1 & 1 & n.d. & n.d. & n.d. & n.d. \\
\hline $\begin{array}{l}\text { Grandes empresas } \\
\text { industriales }\end{array}$ & n.d. & n.d. & n.d. & n.d. & n.d. & n.d. \\
\hline
\end{tabular}

Fuente: Observatorio del Desarrollo de la Universidad de Costa Rica.

En la tabla 2, se muestra claramente una tendencia a la caída en los micro-emprendimientos, especialmente aquellos del área comercial, en los cuales se observa una contracción de 199 empresas desde el año 2003. Igualmente los micro-emprendimientos a nivel industrial son cada vez más reducidos. Finalmente, se puede visualizar que la existencia de grandes empresas en la zona es prácticamente nula.
La problemática de la economía campesina, la vocación forestal de la zona, la degradación de los suelos, la ganadería extensiva y todos los problemas vinculados a un profundo subdesarrollo rural, colocan a este cantón en esta lógica inercial de pauperización y precarización, por ende en una condición de punto muerto en las dinámicas necesarias para crear nuevos empleos.

Cabe resaltar problemas que han empeorado la situación del cantón, el del tabaco, cuya producción quedó desechada por la falta de contratos de compra de las compañías fabricantes de cigarrillos que ante la baja de aranceles trasladaron la producción; el del café con su problema de sobreoferta y bajos precios en el mercado mundial; de la caña que no ha logrado vincularse al mercado mundial; así como el poco potencial turístico y de inversión externa en la zona (Morales, 2007).

Otro asunto de raíz histórica es la tenencia de la tierra, los campesinos venidos del VaIle Central colonizaron la zona e hicieron denuncios de fincas que eran grandes, luego las familias numerosas repartieron las fincas esto provocó un proceso de fraccionamiento de la propiedad poco productivo y poco rentable, por ende a la larga insostenible. Existen muchos pobladores del campo y pocos campesinos, de 38141 habitantes en la zona, sólo 1200 familias cultivan la tierra, esto ha creado desesperanza entre los jóvenes que no ven como opción quedarse en la finca y estudian o buscan empleo, siempre en búsqueda de abandonar la zona. A continuación se puede observar que de la población 61\% del total corresponde a la fuerza de trabajo económicamente activa. 
TABLA 3

\section{PROYECCIÓN DE POBLACIÓN PARA \\ PURISCAL-TURRUBARES (AÑO 2012)}

\begin{tabular}{|c|c|c|c|}
\hline & TOTAL & Hombres & Mujeres \\
\hline Edad & 38141 & 19175 & 18967 \\
\hline 0 & 487 & 260 & 227 \\
\hline 1 & 454 & 250 & 203 \\
\hline 2 & 496 & 271 & 224 \\
\hline 3 & 520 & 273 & 247 \\
\hline 4 & 507 & 272 & 235 \\
\hline 5 & 495 & 263 & 231 \\
\hline 6 & 515 & 283 & 232 \\
\hline 7 & 541 & 281 & 260 \\
\hline 8 & 546 & 302 & 244 \\
\hline 9 & 582 & 303 & 279 \\
\hline 10 & 636 & 324 & 312 \\
\hline 11 & 628 & 333 & 295 \\
\hline 12 & 600 & 293 & 307 \\
\hline 13 & 682 & 340 & 341 \\
\hline 14 & 749 & 378 & 371 \\
\hline $15-19$ & 3884 & 1988 & 1896 \\
\hline $20-24$ & 3767 & 1928 & 1840 \\
\hline $25-29$ & 3019 & 1500 & 1520 \\
\hline $30-34$ & 2465 & 1194 & 1271 \\
\hline $35-39$ & 2288 & 1096 & 1191 \\
\hline $40-44$ & 2718 & 1298 & 1420 \\
\hline $45-49$ & 2611 & 1263 & 1348 \\
\hline $50-54$ & 2249 & 1142 & 1107 \\
\hline $55-59$ & 1730 & 866 & 863 \\
\hline $60-64$ & 1391 & 674 & 717 \\
\hline $65-69$ & 1127 & 553 & 574 \\
\hline $70-74$ & 933 & 477 & 456 \\
\hline $75-79$ & 692 & 338 & 355 \\
\hline $80+$ & 832 & 434 & 398 \\
\hline
\end{tabular}

La marginalidad es más profunda entre mujeres y jóvenes. La disminución paulatina del ingreso monetario en el medio rural se presenta paralela a las presiones del sistema para que le gente consuma. La institucionalidad existente en Puriscal ha fallado en buscar alternativas para el desarrollo del cantón. Finalmente, el aumento de valores de las propiedades a causa de compras de tierras por parte de extranjeros crea un problema inmobiliario, debido a que la zona es una de las más tranquilas y seguras del país.

\section{Estrategia de ecodesarrollo, una posible respuesta para el cantón de Puriscal}

Con el propósito de plantear la estrategia, se debe partir de la realidad del propio cantón y de sus habitantes, desde el enfoque de investigación-acción. Esta metodología considera los conocimientos y la experiencia de los campesinos, qué planes exitosos de sobrevivencia han ejecutado y cómo han enfrentado la degradación de los recursos. Así, partiendo de esa realidad económica, social, ambiental y de otras experiencias globales que aporten los investigadores, se plantean alternativas. Propuestas similares han sido trazadas por Carmen y Sobrado (2002), en diversas partes de Costa Rica y Centroamérica.

El proceso debe movilizar a las fuerzas vivas del cantón, la Unión de Pequeños Productores Agropecuarios, ONG como la Fundación Ecotrópica, instituciones del Estado, la municipalidad y las universidades deben coordinar la estrategia que incluye la búsqueda de alternativas productivas con el apoyo técnico requerido.

Asimismo, se debe recabar la experiencia de los campesinos y movilizar a las personas que residen fuera del cantón, quienes también pueden 
aportar al proceso. Además, se debe incluir una respuesta factible en lo que concerniente a la precariedad geológica de Puriscal.

Por tanto, la estrategia debe estar basada en los campesinos y sus problemáticas, con miras al sector exportador, a rescatar la identidad cultural del cantón, el ecoturismo de base agrícola. Una estrategia de ecodesarrollo a partir de los problemas económicos y ambientales de la región, con el fin de proponer cultivos sustitutos y ganadería alternativa, agroforestería u otras opciones que potencien el desarrollo sostenible y que sean económicamente factibles.

Algunos cuestionamientos también son imprescindibles: ¿está el cantón en posición de aprovechar los nuevos mercados de carbono con actividades agroforestales?, ¿se cuenta con potencial turístico (cómo y en que áreas)?, ¿se puede reactivar la producción alimentaria, con miras a dar respuestas a la crisis?, ¿es posible la vinculación a actividades de exportación?, ¿se puede promover cierto tipo de inversión extranjera en el cantón?, ¿cuáles fuentes de energía se pueden usar?, ¿cuáles posibilidades da la regeneración del bosque producto de la misma crisis agrícola?, ¿cuáles posibilidades tiene la citricultura combinada con café u otros cultivos?, ¿se pueden fortalecer prácticas de conservación de suelos?, entre otras.

La promoción de un enfoque de investigaciónacción, que le brinde alternativas viables a Puriscal, es una necesidad que las universidades públicas debieran considerar como prioritaria.

Las propuestas deben surgir desde la propia realidad socioeconómica y ambiental del cantón sin ignorar el contexto global y nacional, la destrucción de la economía campesina ha sido un plan deliberado del Gobierno y una lógica de la globalización.
Se deben definir políticas claras para el sector campesino, recuperar el suelo, cuidar y cultivar el bosque, visualizar alternativas productivas acordes con la realidad campesina, contar con asistencia técnica y acceso a mercados, entre otros factores clave para el desarrollo.

Dentro de este contexto, ¿es la apertura y la liberalización incondicional un camino para los campesinos?, ¿es la crisis global alimentaria y la misma crisis ambiental una indicación del camino por seguir?; no se debe obviar que la organización y la lucha es clave en Europa, Japón y EE.UU., países donde la producción agraria está muy protegida ¿cómo competir entonces con esos mercados?

\section{CONCLUSIONES Y RECOMENDACIONES}

Las limitantes de desarrollo regional, a lo interno de los países, permiten explicar el porqué existen zonas rezagadas en el proceso de crecimiento de un país. En el caso del cantón de Puriscal se visualizan problemáticas de raíz ambiental, relacionadas con no utilizar los terrenos para su vocación natural y otro tipo de situaciones que provocan una serie de dificultades socioeconómicas que obstaculizan el desarrollo de la zona.

Por su parte, la esperanza del cantón siempre estará en la capacidad creativa de sus campesinos, quienes en algunos casos han diversificado la producción: ganadería semiestabulada para liberar tierras, abonos verdes para eliminar la dependencia de insumos, parches de bosque, empresas de agroecoturismo campesino, modalidades que prometen mejorar la calidad de vida y recuperar los ecosistemas del cantón.

Las acciones deben ejecutarse bajo la coordinación interinstitucional e intersectorial, ya que 
en todas las instituciones hay carencias que pueden solventarse mediante el trabajo en equipo, aprovechando las potencialidades de cada una y complementando las necesidades que surjan.

Se debe fortalecer la información, la capacitación efectiva y el seguimiento a las iniciativas de desarrollo, además del estímulo por parte de las instituciones y organizaciones locales, regional y nacionales, mediante propuestas técnicas y financieras que busquen atraer fondos para el desarrollo del cantón.

Igual de importante es el financiar investigaciones que ilustren los procesos descritos, construyan indicadores para evaluarlos y observar su evolución, y además midan el impacto de las acciones propuestas.

Se espera que los resultados de este estudio contribuyan a la necesaria crítica por parte de la UNED del modelo de desarrollo seguido en el país y a la búsqueda de alternativas para el cantón de Puriscal; como también a las instituciones interesadas en planificar y ejecutar políticas de desarrollo en la zona, pues con el apoyo y proyección requeridos pueden surgir insumos de capacitación, investigación, extensión y desarrollo regionales necesarios.

De esta forma, la investigación podría aportar insumos relevantes para la elaboración de políticas institucionales y estatales que permitan dinamizar la cantidad y calidad de empleos en la zona, comprender las raíces de la crisis del

sector agrícola en la región mediante un conocimiento objetivo de las características y del comportamiento del mercado laboral.

La Universidad Estatal a Distancia debe potenciar un nuevo papel del centro universitario como gestor del desarrollo regional, lo cual se convierte en una necesidad cada vez mayor dadas las nuevas políticas y los procesos de regionalización y desconcentración en boga en la institución.

\section{Referencias}

Carmen, R. y Sobrado, M. (Ed) (2002). Un futuro para los excluidos: Creación de Empleos y Generación de Ingreso por los hombres: Clodomir Santos de Morais y el Laboratorio Organizacional .Heredia: Editorial Universidad Nacional.

Cervantes, C. y Gunther V. W. (1992). Características físicas y pérdida de nutrientes de las parcelas de erosión de Cerbatana de Puriscal, Costa Rica. Revista Agronomía Costarricense,16(1), 99-106.

Duchrow U. y Hinkelammert, F. ( 2004). Apuntes y esbozos para la presentación del Seminario "Vida o Capital" en el marco del SIF, DEI, Costa Rica, 30 de agosto -3 de septiembre del 2004.

Hinkelamert, Franz (1983). Dialéctica del Desarrollo Desigual. Colección Aula. San José, Costa Rica. Universidad Centroamericana. Educa. Recuperado de

http://www.scielo.org.ve/scielo.php?script=sci_ arttext\&pid=S1315-85972006000200003\&lng=es\&n $r m=i s o$

Linares S, J. (2006). Cambios en las relaciones laborales y nuevas formas de organización. Gaceta Laboral 12 (2).

Marín, E, y Vega, M. (2000). La reforma laboral en América Latina. Un análisis comparado. Lima OIT/ETM. Recuperado de http://www.cinterfor.org.uy/public/ spanish/region/ampro/cinterfor/newsroom/resenas/2000/mayo/reflab.htm

Montoya, F. (2004). . Recuperado de: http://www.odd.ucr. ac.cr/phocadownload/programa-investigacion-ensinecologia.pdf

Morales Hernández, F (2007-19 de febrero). . Diario Extra pag. 3. Recuperado de: http://www.ministrabajo. go.cr/Ministro/ARTICULOS/coopepuriscal.htm

Neffa, J. C. ( 2006). Fondo de Cultura Económica, Buenos Aires. Recuperado de: http://www.ceil-piette.gov.ar/ docpub/libros/mercados1.htm 
RNA Revista Nacional de Administración

Programa de Naciones Unidas para el Desarrollo y Universidad de Costa Rica (2011). , . Recuperado de: http:// www.pnud.or.cr/mapa-cantonal/

Somarribas Chacón, Leonel y Patterson, Ora (2008). . Recuperado de: http://www.una.ac.cr/geog/Revista/ Revcontenido.htm

Sommarribas, L. y Patterson, O. (1997). Degradación de recursos, pobreza y salud en el cantón de Puriscal. Revista Geográfica de América Central, N 34, II semestre 1996- I semestre 1997, pp. 57-85. Recuperado de: http://www.revistas.una.ac.cr/index.php/geografica/ article/view/1897

Universidad Estatal a Distancia. Plan de desarrollo institucional: para el fortalecimiento de la educación a distancia. Sabanilla. EUNED. Aprobado por el Consejo Universitario, sesión 2001-2011.

Recibido: 13 de octubre de 2012 Aceptado: 3 de febrero de 2013 